\title{
Review of: "Efficacy, safety, and lot to lot immunogenicity of an inactivated SARS-CoV-2 vaccine (BBV152): a double-blind, randomised, controlled phase 3 trial"
}

\author{
Arindam Basu ${ }^{1}$ \\ 1 University of Canterbury
}

Potential competing interests: The author(s) declared that no potential competing interests exist.

This is a preprint version of a phase III randomised double blind trial of the inactivated sars-cov- 2 vaccine (BBV152) in an Indian population of about 19, 000 people conducted over 52 days. The trial results report a $77 \%$ vaccine efficacy with no significant adverse effects attributable to the vaccine itself. A few points worth noting:

1. The sample size was based on finding 130 individuals who were enrolled in the trial to have COVID19; in estimating the sample size, they assumed a 1\% attack rate among the unvaccinated (or placebo), and a $60 \%$ Vaccine Efficacy with 95\% confidence interval. When these figures were entered in the WHO sample size calculator for vaccine efficacy studies ${ }^{[1]}$, it turned out that the study would need 86,000 participants. By that standard, the study was underpowered

2. While the study was designed to be placebo controlled double blind studies, it relied on voluntary participation of people, and it was not clear how participants in the study were recruited, i.e., from what sources or how did they obtain the participants. As a result, a self-reporting bias could not be ruled out.

3. While the study employed masking and blinding of the participants and "those associated" with the study, the study data analysts were unblinded and therefore risks of biases in the analyses of the data could not be ruled out

4. For any RCT, external validity is an issue [2], and whether the results obtained in this phase III trial can be widely applied to others and for prevention of newer variants of concern is not clear.

5. An average efficacy of $77 \%$ for symptomatic COVID19 for those who are fully vaccinated (i.e., 2 weeks after two completed doses and continuously monitored) is about $14 \%$ lower than the mRNA vaccine (Pfizer-Biontec) as reported by The CDC (see https://www.cdc.gov/coronavirus/2019ncov/vaccines/different-vaccines/Pfizer-BioNTech.html)

6. Problems with allocation concealment is a known problem in clinical trials [3]; while the article stated that they had adopted "allocation concealment", how that was achieved was not clearly stated in the methods section

7. This clinical trial adopted a per protocol analysis of the results. This can lead to several problems including those of biased analyses, and reduction in power, as described previously [4] 
Overall, while this double blind placebo controlled efficacy trial of an inactivated vaccine suggest that the vaccine itself is about $77 \%$ effiacious in preventing new infections from alpha variant, a few points need to be kept in mind - that such protection may be inadequate particularly in the face of emergence of new variants; the study was also conducted in India at a time when the alpha variant infections were on a decline, so the effects may have been overestimated. The study itself appears to be underpowered and open to several biases.

\section{References}

1. `WHO. (2021). Sample size calculator for evaluation of COVID-19 vaccine effectiveness (Excel). WHO.

2. ^Peter M Rothwell. (2006). Factors That Can Affect the External Validity of Randomised Controlled Trials. PLOS Clin Trial, vol. 1 (1), e9. doi:10.1371/journal.pctr.0010009.

3. `Catherine L Hill, Michael P LaValley, David T Felson. (2002). Discrepancy between published report and actual conduct of randomized clinical trials. Journal of Clinical Epidemiology, vol. 55 (8), 783-786. doi:10.1016/s0895-4356(02)00440-7.

4. ^Priya Ranganathan, CS Pramesh, Rakesh Aggarwal. (2016). Common pitfalls in statistical analysis: Intention-to-treat versus per-protocol analysis. Perspect Clin Res, vol. 7 (3), 144. doi:10.4103/22293485.184823. 